H. Hashimoto and K. Mashimo

Nagoya Math. J.

Vol. 156 (1999), 171-185

\title{
ON SOME 3-DIMENSIONAL CR SUBMANIFOLDS IN $S^{6}$
}

\author{
HIDEYA HASHIMOTO AND KATSUYA MASHIMO
}

\author{
Dedicated to Professor Tsunero Takahashi \\ on his sixtieth birthday
}

\begin{abstract}
We give two types of 3-dimensional CR-submanifolds of the 6dimensional sphere. First we study whether there exists a 3-dimensinal CRsubmanifold which is obtained as an orbit of a 3-dimensional simple Lie subgroup of $G_{2}$. There exists a unique (up to $G_{2}$ ) 3-dimensional CR-submanifold which is obtained as an orbit of reducible representations of $S U(2)$ on $\mathbf{R}^{7}$. As orbits of the subgroup which corresponds to the irreducible representation of $S U(2)$ on $\mathbf{R}^{7}$, we obtained 2-parameter family of 3-dimensional CRsubmanifolds. Next we give a generalization of the example which was obtained by K. Sekigawa.
\end{abstract}

\section{Introduction}

Let $(M, J,\langle\rangle$,$) be an almost Hermitian manifold. For a submanifold N$ of $M$, we put $\mathcal{H}_{x}=T_{x} N \cap J\left(T_{x} N\right)(x \in N)$ and denote by $\mathcal{H}_{x}^{\perp}$ the orthogonal complement of $\mathcal{H}_{x}$ in $T_{x} N$. If the dimension of $\mathcal{H}_{x}$ is constant and $J\left(\mathcal{H}_{x}^{\perp}\right) \subset T_{x}^{\perp} N$ for any $x \in N$, the submanifold $N$ is called a $C R$ submanifold. Especially if $\mathcal{H}_{x}=T_{x} N$, the submanifold $N$ is said to be a holomorphic (or invariant) submanifold and if $\operatorname{dim}\left(\mathcal{H}_{x}\right)=0$ and $J\left(T_{x} N\right) \subset T_{x}^{\perp} N$ for any $x \in N$, the submanifold $N$ is said to be a totally real submanifold.

It is well-known that the 6-dimensional sphere $S^{6}$ admits an almost complex structure. On the existence of holomorphic or totally real submanifold of $S^{6}$, many results are obtained. A. Gray proved that there does not exist any 4-dimensional holomorphic submanifold ([7]) and R. Bryant proved that there exist infinitely many 2 -dimensional holomorphic submanifolds ([1]). It was proved by Ejiri that any 3-dimensional totally real sub-

Received May 12, 1997.

The authors are partially supported by Grants-in-Aid for Scientific Research, The Ministry of Culture and Education, Japan 
maifold of $S^{6}$ is a minimal submanifold ([4]). He also proved that some tubes in the direction of the first and the second normal bundle of holomorphic curves are totally real submanifolds of $S^{6}([5])$. The second author classified 3-dimensional homogeneous minimal submanifolds of $S^{6}$ and determined all 3-dimensional homogeneous totally real submanifolds of $S^{6}$ ([11]).

Though there are many results on the existence of holomorphic submanifolds and totally real submanifolds of $S^{6}$, only one example is known about the existence of CR submanifold of $S^{6}$ ([13]).

The aim of this paper is to give many 3-dimensional CR submanifolds of $S^{6}$ with $\operatorname{dim}_{\mathbf{R}} \mathcal{H}=2$. Second author proved that a 3 -dimensional subspace $V$ in $\mathbf{C}^{3}$ satisfies $\operatorname{dim}_{\mathbf{R}}(V \cap J(V))=2$ if and only if $\omega(V)=0$, where $J$ is the complex structure and $\omega$ is the Laglangean 3 -form. The fact is also used in this paper.

\section{$\S 1$. Preliminaries}

\subsection{Cayley algebra}

Let $\mathbf{H}$ be the skew field of all quaternions. The Cayley algebra $\mathfrak{C}$ over $\mathbf{R}$ is $\mathfrak{C}=\mathbf{H} \oplus \mathbf{H}$ with the following multiplication;

$$
(q, r) \cdot(s, t)=(q s-\bar{t} r, t q+r \bar{s}), \quad q, r, s, t \in \mathbf{H}
$$

where " $"$ means the conjugation in $\mathbf{H}$. We define a conjugation in $\mathfrak{C}$ by $\overline{(q, r)}=(\bar{q},-r), q, r \in \mathbf{H}$, and an inner product $\langle$,$\rangle by$

$$
\langle x, y\rangle=(x \cdot \bar{y}+y \cdot \bar{x}) / 2, \quad x, y \in \mathfrak{C} .
$$

We put

$$
\mathfrak{C}_{0}=\{x \in \mathfrak{C} \mid x+\bar{x}=0\} .
$$

The Cayley algebra $\mathfrak{C}$ is neither commutative nor associative. But we have the following

(1) If $x, y \in \mathfrak{C}_{0}$, then $x \cdot y=-y \cdot x$.

(2) For any $x, y, z \in \mathfrak{C}$,

$$
\bar{x} \cdot(x \cdot y)=(\bar{x} \cdot x) \cdot y, \quad\langle x \cdot y, x \cdot z\rangle=\langle x, x\rangle\langle y, z\rangle .
$$

(3) If $x, y, z \in \mathfrak{C}$ are mutually orthogonal unit vectors,

$$
x \cdot(y \cdot z)=y \cdot(z \cdot x)=z \cdot(x \cdot y) .
$$


The unit sphere $S^{6} \subset \mathfrak{C}_{0}$ centered at the origin has an almost complex structure $J$ defined by

$$
J_{p}(X)=p \cdot X \quad p \in S^{6}, X \in T_{p} S^{6} .
$$

We use the canonical orthonormal basis $e_{0}=(1,0), e_{1}=(i, 0), e_{2}=$ $(j, 0), e_{3}=(k, 0), e_{4}=(0,1), e_{5}=(0, i), e_{6}=(0, j), e_{7}=(0, k)$ of the Cayely algebra, where $1, i, j, k$ is the standard orthonormal basis of $\mathbf{H}$. The vector $e_{0}$ is the unit element of $\mathfrak{C}$ and the product $e_{i} \cdot e_{j}$ is given in the following table;

\begin{tabular}{|c||r|r|r|r|r|r|r|}
\hline$i \backslash j$ & 1 & 2 & 3 & 4 & 5 & 6 & 7 \\
\hline 1 & $-e_{0}$ & $e_{3}$ & $-e_{2}$ & $e_{5}$ & $-e_{4}$ & $-e_{7}$ & $e_{6}$ \\
2 & $-e_{3}$ & $-e_{0}$ & $e_{1}$ & $e_{6}$ & $e_{7}$ & $-e_{4}$ & $-e_{5}$ \\
3 & $e_{2}$ & $-e_{1}$ & $-e_{0}$ & $e_{7}$ & $-e_{6}$ & $e_{5}$ & $-e_{4}$ \\
4 & $-e_{5}$ & $-e_{6}$ & $-e_{7}$ & $-e_{0}$ & $e_{1}$ & $e_{2}$ & $e_{3}$ \\
5 & $e_{4}$ & $-e_{7}$ & $e_{6}$ & $-e_{1}$ & $-e_{0}$ & $-e_{3}$ & $e_{2}$ \\
6 & $e_{7}$ & $e_{4}$ & $-e_{5}$ & $-e_{2}$ & $e_{3}$ & $-e_{0}$ & $-e_{1}$ \\
7 & $-e_{6}$ & $e_{5}$ & $e_{4}$ & $-e_{3}$ & $-e_{2}$ & $e_{1}$ & $-e_{0}$ \\
\hline
\end{tabular}

\subsection{Exceptional simple Lie group $G_{2}$}

It is well-known that the group of all automorphisms of $\mathfrak{C}$ is a compact connected simple Lie group of type $\mathfrak{g}_{2}([6])$, which we denote by $G_{2}$. The group $G_{2}$ leaves the vector $e_{0}$ and the subspace $\mathfrak{C}_{0}=\sum_{i=1}^{7} \mathbf{R} e_{i}$ invariant. Furthermore $G_{2}$ leaves the inner product $\langle$,$\rangle invariant. If we identify \mathfrak{C}_{0}$ with the set of all 7-dimensional column vectors in a natural manner, then $G_{2}$ is a subgroup of $S O(7)$.

LEMMA 1. For a pair of mutually orthogonal unit vectors $a_{4}, a_{1}$ in $\mathfrak{C}_{0}$ put $a_{5}=a_{1} \cdot a_{4}$. Take a unit vector $a_{2}$, which is perpendicular to $a_{4}, a_{1}$ and $a_{5}$. If we put $a_{3}=a_{1} \cdot a_{2}, a_{6}=a_{2} \cdot a_{4}$ and $a_{7}=a_{3} \cdot a_{4}$ then the matrix

$$
g=\left(a_{1}, a_{2}, a_{3}, a_{4}, a_{5}, a_{6}, a_{7}\right) \in S O(7)
$$

is an element of $G_{2}$ with $g \cdot e_{4}=a_{4}$.

For the proof of Lemma 1, we refer to [8]. 
Let $G_{i j}(1 \leq i \neq j \leq 7)$ be the skew symmetric transformation on $\mathfrak{C}_{0}$ defined by

$$
G_{i j}\left(e_{k}\right)=\left\{\begin{aligned}
e_{i}, & \text { if } k=j \\
-e_{j}, & \text { if } k=i \\
0, & \text { otherwise }
\end{aligned}\right.
$$

The Lie algebra $\mathfrak{g}_{2}$ of $G_{2}$ is spanned by the following vectors in the Lie algebra $\mathfrak{s o}(7)$ of $S O(7)$;

$$
\left\{\begin{array}{l}
a G_{23}+b G_{45}+c G_{76}, \\
a G_{31}+b G_{46}+c G_{57}, \\
a G_{12}+b G_{47}+c G_{65}, \\
a G_{51}+b G_{73}+c G_{62}, \\
a G_{14}+b G_{72}+c G_{36}, \\
a G_{17}+b G_{24}+c G_{53}, \\
a G_{61}+b G_{34}+c G_{25}
\end{array}\right.
$$

where $a, b, c$ are real numbers with $a+b+c=0$.

\subsection{A criterion for a CR subspace}

Let $J$ be the standard complex structure on $\mathbf{C}^{3}$ with the standard Hermitian metric. Take an orthonormal basis $e_{1}, e_{2}, e_{3}, e_{4}=J\left(e_{1}\right), e_{5}=$ $J\left(e_{2}\right), e_{6}=J\left(e_{3}\right)$ of $\mathbf{C}^{3}$. We denote by $\omega_{1}, \cdots, \omega_{6}$ the orthonormal coframe on $\mathbf{C}^{3}$ dual to $e_{1}, \cdots, e_{6}$. Put

$$
\omega=\left(\omega_{1}+\sqrt{-1} \omega_{4}\right) \wedge\left(\omega_{2}+\sqrt{-1} \omega_{5}\right) \wedge\left(\omega_{3}+\sqrt{-1} \omega_{6}\right)
$$

Remember that $\omega$ depends on the choise of the basis $e_{1}, \cdots, e_{6}$. For an element $g \in U(3)$ we have

$$
g^{*} \omega=\operatorname{det}(g) \omega .
$$

Proposition 2. A 3-dimensional real subspace $V$ of $\mathbf{C}^{3}$ satisfies $\operatorname{dim}_{\mathbf{R}}(V \cap J(V))=2$ if and only if $\omega(V)=0$.

If a 3-dimensional real subspace $V$ of $\mathbf{C}^{3}$ satisfies $\operatorname{dim}_{\mathbf{R}}(V \cap J(V))=2$ then it also satisfies $J\left((V \cap J V)^{\perp} \cap V\right) \subset V^{\perp}$. For a 3-dimensional CR submanifold of a 6-dimensional almost complex manifold which is not a totally real submanifold we have $\operatorname{dim}_{\mathbf{R}}\left(T_{x} N \cap J\left(T_{x} N\right)\right)=2$. Thus we have the following

Corollary 3. Let $M$ be a 6-dimensional almost complex manifold. A 3-dimensional submanifold $N$ of $M$ is a CR submanifold with $\operatorname{dim} \mathcal{H}=2$ if and only if $\omega\left(T_{x} N\right)=0$ for any $x \in N$. 


\section{$\S 2$. Orbits of TDS in $G_{2}$}

In this section, we study 3-dimensional CR submanifolds which are orbits of some 3-dimensional simple subgroup (abbreviated as TDS) of $G_{2}$.

\subsection{Classification of TDS in $G_{2}$}

Let $\mathfrak{g}$ be a compact simple Lie algebra and $\mathfrak{t}$ be a maximal abelian subalgebra of $\mathfrak{g}$. Let $\mathfrak{u}$ be a simple 3 -dimensional subalgebra of $\mathfrak{g}$. Take a basis $X_{1}, X_{2}, X_{3}$ of $\mathfrak{u}$ with

$$
\left[X_{1}, X_{2}\right]=2 X_{3},\left[X_{2}, X_{3}\right]=2 X_{1},\left[X_{3}, X_{1}\right]=2 X_{2}
$$

and put

$$
\left\{\begin{array}{l}
H=\sqrt{-1} X_{1}, \\
X_{+}=(1 / \sqrt{2})\left(X_{2}+\sqrt{-1} X_{3}\right), \\
X_{-}=(1 / \sqrt{2})\left(-X_{2}+\sqrt{-1} X_{3}\right) .
\end{array}\right.
$$

The bracket products of the basis $H, X_{+}, X_{-}$of $\mathfrak{u}^{\mathbf{C}}$ are

$$
\left[H, X_{+}\right]=2 X_{+},\left[H, X_{-}\right]=-2 X_{-},\left[X_{+}, X_{-}\right]=H
$$

We may assume that $H$ is contained in $\sqrt{-1} \mathrm{t}$. Hence $\alpha(H)$ is a real number for every root $\alpha$ of $\mathfrak{g}^{\mathbf{C}}$ with respect to $\mathfrak{t}^{\mathbf{C}}$. Furthermore $\alpha(H)=0,1$ or 2 if $\alpha$ is a simple root ([3, p.166]). The weighted Dynkin diagram with weight $\alpha(H)$ added to each vertex $\alpha$ of the Dynkin diagram of $\mathfrak{g}^{\mathbf{C}}$ is called the characteristic diagram of $\mathfrak{u}$. Let $\mathfrak{u}$ and $\mathfrak{u}^{\prime}$ be 3 -dimensional simple Lie subalgebras of $\mathfrak{g}$. Then $\mathfrak{u}$ and $\mathfrak{u}^{\prime}$ are mutually conjugate in $\mathfrak{g}$ if and only if $\mathfrak{u}^{\mathbf{C}}$ and $\mathfrak{u}^{\prime \mathbf{C}}$ have the same characteristic diagram.

Mal'cev [10] classified the 3-dimensional complex simple subalgebras of $\mathfrak{g}_{2}^{\mathbf{C}}$. From his classification, $\mathfrak{g}_{2}$ has 4 types of 3 -dimensional simple subalgebras.

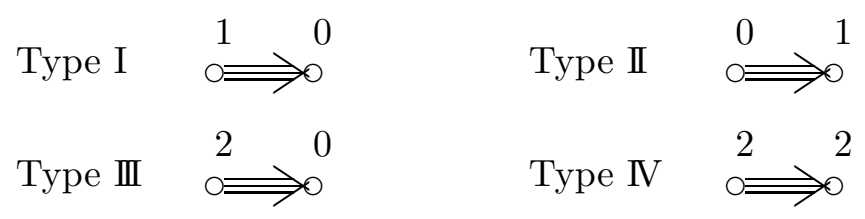

We shall study 3 dimensional homogeneous CR submanifolds of $S^{6}$ which are orbits of 3 dimensional simple Lie subgroup of $G_{2}$. We denote by $\omega_{i}$ the orthogonal coframes on $\mathfrak{C}_{0}$ dual to $e_{i}$. We also denote by $\omega_{i}$ the 
restriction of $\omega_{i}$ to $S^{6}$. Since $J_{e_{4}}\left(e_{1}\right)=-e_{5}, J_{e_{4}}\left(e_{2}\right)=-e_{6}$ and $J_{e_{4}}\left(e_{3}\right)=$ $-e_{7}$, we have

$$
\omega_{\mid e_{4}}=\left(\omega_{1}-\sqrt{-1} \omega_{5}\right) \wedge\left(\omega_{2}-\sqrt{-1} \omega_{6}\right) \wedge\left(\omega_{3}-\sqrt{-1} \omega_{7}\right) .
$$

\subsection{Orbit of the TDS of type I}

A basis of the subalgebra with (1) corresponding to the characteristic diagram of type I is as follows;

$$
\left\{\begin{array}{l}
X_{1}=-G_{45}+G_{76}, \\
X_{2}=-G_{46}+G_{57}, \\
X_{3}=-G_{47}+G_{65} .
\end{array}\right.
$$

We denote by $U_{1}$ the Lie subgroup of $G_{2}$ generated by the subalgebra. The subgroup $U_{1}$ is isomorphic to $S p(1)$ and acts on $\mathfrak{C}_{0}$ as follows;

$$
q \cdot(x, y)=(x, y \bar{q}), \quad q \in S p(1) .
$$

In this case, $\mathbf{R} e_{1}, \mathbf{R} e_{2}, \mathbf{R} e_{3}$ and $\sum_{j=4}^{7} \mathbf{R} e_{j}$ are invariant irreducible subspaces so that each orbit is a small sphere or a great sphere.

\subsection{Orbit of the TDS of type II}

A basis satisfying (1) of the subalgebra corresponding to the characteristic diagram of type II is as follows;

$$
\left\{\begin{array}{l}
X_{1}=-2 G_{23}+G_{45}+G_{76}, \\
X_{2}=-2 G_{31}+G_{46}+G_{57}, \\
X_{3}=-2 G_{12}+G_{47}+G_{65} .
\end{array}\right.
$$

We denote by $U_{2}$ the Lie subgroup of $G_{2}$ generated by the subalgebra. The subgroup $U_{2}$ is isomorphic to $S p(1)$ and acts on $\mathfrak{C}_{0}$ as follows;

$$
q \cdot(x, y)=(q x \bar{q}, y \bar{q}), \quad q \in S p(1) .
$$

THEOREM 4. Let $N$ be the orbit of $U_{2}$ through the point $p_{0}=(1 / 3) e_{2}+$ $(2 \sqrt{2} / 3) e_{4}$. Any 3 dimensional CR submanifold of $S^{6}$, which is an orbit of $U_{2}$ in $S^{6}$, is congruent to $N$ under the action of $G_{2}$ on $S^{6}$.

Proof. Take a point $p$ on $S^{6}$ and consider the orbit $M=U_{2} \cdot p$ of $U_{2}$ through $p$. Since the action of $S p(1)$ on $S^{3} \subset H$ by $y \rightarrow y \bar{q} \quad(q \in S p(1))$ is transitive, we may assume that $p$ is of the form $p=\sum_{i=1}^{4} x_{i} e_{i}$. Put

$$
g_{t}=\exp \left(t\left(X_{3}-\left(G_{47}-G_{65}\right)\right)\right)=\exp \left(-2 t\left(G_{12}-G_{65}\right)\right)
$$


and consider the one parameter subgroup $Z=\left\{g_{t}: t \in \mathbf{R}\right\}$. Since $G_{47}-G_{65}$ commutes with $X_{1}, X_{2}$ and $X_{3}$ we have

$$
U_{2} \cdot g_{t} \cdot p=g_{t} \cdot M
$$

Namely the orbit $M$ is congruent to the orbit through $p^{\prime}=\sum_{i=2}^{4} x_{i} e_{i}$. If $x_{4}=0$ then we have $\operatorname{dim}(M)=2$. Thus we assume $x_{4} \neq 0$.

Put $a_{4}=p^{\prime}, a_{1}=e_{6}$ and $a_{5}=a_{1} \cdot a_{4}$. The vector $a_{2}=c\left(x_{4} e_{1}+x_{2} e_{7}\right)$ $\left(c=1 / \sqrt{x_{2}^{2}+x_{4}^{2}}\right)$ is orthogonal to $a_{4}, a_{1}$ and $a_{5}$. Thus by Lemma 1 , the matrix

$$
g=\left(\begin{array}{ccccccc}
0 & 0 & 0 & 0 & 0 & 1 & 0 \\
c x_{4} & 0 & 0 & 0 & 0 & 0 & c x_{2} \\
-c x_{2} & 0 & 0 & 0 & 0 & 0 & c x_{4} \\
0 & x_{2} & x_{3} & x_{4} & 0 & 0 & 0 \\
0 & -x_{4} & 0 & x_{2} & -x_{3} & 0 & 0 \\
0 & -c x_{3} x_{4} & 0 & c x_{2} x_{3} & 1 / c & 0 & 0 \\
0 & c x_{2} x_{3} & -1 / c & c x_{3} x_{4} & 0 & 0 & 0
\end{array}\right)
$$

is an element of $G_{2}$ with $g \cdot p^{\prime}=e_{4}$.

Substitute

$$
\begin{aligned}
& v_{1}=g_{*}\left(X_{1}\left(p^{\prime}\right)\right)=\left(3 x_{3} x_{4}\right) e_{5}+c x_{4}\left(3 x_{3}^{2}-1\right) e_{6}-2 c x_{2} e_{7}, \\
& v_{2}=g_{*}\left(X_{2}\left(p^{\prime}\right)\right)=-x_{4} e_{1}+\left(2 c x_{3} x_{4}\right) e_{2}-\left(2 c x_{2} x_{3}\right) e_{3} \\
& v_{3}=g_{*}\left(X_{3}\left(p^{\prime}\right)\right)=-3 c x_{2} x_{4} e_{2}+c\left(2 x_{2}^{2}-x_{4}^{2}\right) e_{3}
\end{aligned}
$$

into $\left.\omega\right|_{e_{4}}$, we have

$$
\left.\omega\right|_{e_{4}}\left(v_{1}, v_{2}, v_{3}\right)=\sqrt{-1} c^{2} x_{4}^{2}\left(8 x_{2}^{2}+x_{4}^{2}\left(9 x_{3}^{2}-1\right)\right) .
$$

Thus the orbit $M=U_{2}\left(p^{\prime}\right)$ through the point $p^{\prime}=x_{2} e_{2}+x_{3} e_{3}+x_{4} e_{4}$ is a 3-dimensional CR submanifold of $S^{6}$ if and only if

$$
\left\{\begin{array}{l}
x_{4} \neq 0 \\
x_{2}^{2}+x_{3}^{2}+x_{4}^{2}=1 \\
8 x_{2}^{2}+x_{4}^{2}\left(9 x_{3}^{2}-1\right)=0 .
\end{array}\right.
$$

The solution of the above equations is as follows;

$$
x_{2}^{2}+x_{3}^{2}=1 / 9, x_{4}^{2}=8 / 9 .
$$

Every orbit through a point which satisfies (3) is congruent to $N$ by $\exp \left(t\left(G_{23}-G_{76}\right)\right) \in G_{2}$ for some $t \in \mathbf{R}$. 


\subsection{Orbit of the TDS of type III}

A basis satisfying (1) of the subalgebra corresponding to the characteristic diagram of type III is as follows;

$$
\left\{\begin{array}{l}
X_{1}=-2 G_{21}-2 G_{65}, \\
X_{2}=-2 G_{32}-2 G_{76}, \\
X_{3}=-2 G_{31}-2 G_{75}
\end{array}\right.
$$

We denote by $U_{3}$ the Lie subgroup of $G_{2}$ generated by the subalgebra. The subgroup $U_{3}$ is isomorphic to $S O(3)$ and the covering group $S p(1)$ of $U_{3}$ acts on $\mathfrak{C}_{0}$ as follows;

$$
q \cdot(x, y)=(q x \bar{q}, q y \bar{q}), \quad q \in S p(1)
$$

THEOREM 5. There does not exist any 3 dimensional CR submanifold of $S^{6}$ which is an orbit of the subgroup $U_{3}$.

Proof. Take a point $p$ on $S^{6}$ and consider the orbit $M=U_{3} \cdot p$ of $U_{3}$ through $p$. Since the action of $S p(1)$ on $S^{2}$ by $x \rightarrow q x \bar{q} \quad(q \in S p(1))$ is transitive, we may assume that $p$ is of the form $p=x_{1} e_{1}+x_{4} e_{4}+x_{5} e_{5}+x_{6} e_{6}$. Put $a_{4}=p, a_{1}=e_{7}$ and $a_{5}=a_{1} \cdot a_{4}$. If $x_{1}=0$ then we have $\operatorname{dim}(M)=2$. Thus we assume $x_{1} \neq 0$. The vector $a_{2}=c\left(x_{4} e_{1}+x_{6} e_{3}-x_{1} e_{4}\right) \quad(c=$ $\left.1 / \sqrt{x_{1}^{2}+x_{4}^{2}+x_{6}^{2}}\right)$ is orthogonal to $a_{4}, a_{1}$ and $a_{5}$. Thus by Lemma 1 , the matrix

$$
g=\left(\begin{array}{ccccccc}
0 & 0 & 0 & 0 & 0 & 0 & 1 \\
c x_{4} & 0 & c x_{6} & -c x_{1} & 0 & 0 & 0 \\
0 & 0 & c x_{1} & c x_{6} & 0 & -c x_{4} & 0 \\
x_{1} & 0 & 0 & x_{4} & x_{5} & x_{6} & 0 \\
x_{6} & -x_{5} & -x_{4} & 0 & 0 & -x_{1} & 0 \\
-c x_{1} x_{5} & 0 & 0 & -c x_{4} x_{5} & 1 / c & -c x_{5} x_{6} & 0 \\
c x_{5} x_{6} & 1 / c & -c x_{4} x_{5} & 0 & 0 & -c x_{1} x_{5} & 0
\end{array}\right)
$$

is an element of $G_{2}$ with $g \cdot p=e_{4}$. Substitute

$$
\begin{aligned}
& v_{1}=g_{*}\left(X_{1}(p)\right)=\left(2 x_{6}, 0,0,0,0,0,0\right), \\
& v_{2}=g_{*}\left(X_{2}(p)\right)=\left(-2 x_{5},-2 c x_{1} x_{6},-2 c x_{1}^{2}, 0,2 x_{1} x_{4}, 0,2 c x_{1} x_{4} x_{5}\right), \\
& v_{3}=g_{*}\left(X_{3}(p)\right)=\left(0,0,-2 c x_{4} x_{5}, 0,-4 x_{1} x_{5},-2 c x_{6}, 2 c x_{1}\left(1-2 x_{5}^{2}\right)\right),
\end{aligned}
$$

into $\left.\omega\right|_{e_{4}}$, we have

$$
\left.\omega\right|_{e_{4}}\left(v_{1}, v_{2}, v_{3}\right)=16 c^{2} x_{1}^{2} x_{6}^{2} \sqrt{-1}\left(1-x_{5}^{2}\right) .
$$


If we assume $\omega\left(v_{1}, v_{2}, v_{3}\right)=0$, we have $x_{1}=0, x_{6}=0$ or $x_{5}= \pm 1$. In any case, the dimension of the orbit is equal to 2 . Thus there does not exist any 3 dimensional orbit which is a CR submanifold of $S^{6}$.

\subsection{Orbit of the TDS of type IV}

A basis satisfying (1) of the subalgebra corresponding to the characteristic diagram of type IV is as follows;

$$
\left\{\begin{array}{l}
X_{1}=4 G_{32}+2 G_{54}+6 G_{76}, \\
X_{2}=\sqrt{6}\left(G_{37}+G_{26}-2 G_{15}\right)+\sqrt{10}\left(G_{42}-G_{35}\right), \\
X_{3}=\sqrt{6}\left(G_{63}+G_{27}-2 G_{41}\right)+\sqrt{10}\left(G_{25}-G_{34}\right) .
\end{array}\right.
$$

We denote by $U_{4}$ the Lie subgroup of $G_{2}$ generated by the subalgebra. The subgroup $U_{4}$ is isomorphic to $S O(3)$.

From Lemma 1 in [2], the linear subspace $\left(\left(\mathbf{R} X_{1}+\mathbf{R} X_{2}+\mathbf{R} X_{3}\right) e_{7}\right)^{\perp}$ meets every orbit of the action of $U_{4}$ on $\mathfrak{C}_{0}$. So the great sphere $S^{3}=$ $\left\{x_{1} e_{1}+x_{4} e_{4}+x_{5} e_{5}+x_{7} e_{7}: x_{1}, x_{4}, x_{5}, x_{7} \in \mathbf{R}\right\} \cap S^{6}$ meets every orbit of the action of $U_{4}$ on $S^{6}$.

THEOREM 6. If the dimension of the orbit $N=U_{4} \cdot p$ through a point $p$ of the great sphere

$$
\left\{x_{1} e_{1}+x_{4} e_{4}+x_{5} e_{5}+x_{7} e_{7}: x_{1}, x_{4}, x_{5}, x_{7} \in \mathbf{R}\right\} \cap S^{6}
$$

is 3 , then it is a CR-submanifold if and only if $f\left(x_{1}, x_{4}, x_{5}, x_{7}\right)=0$ where

$$
\begin{aligned}
f\left(x_{1}, x_{4}, x_{5}, x_{7}\right)= & -5 x_{4}^{4}-10 x_{4}^{2} x_{5}^{2}-5 x_{5}^{4}+42 x_{4}^{2} x_{7}^{2}+72 x_{1}^{2} x_{7}^{2}+42 x_{5}^{2} x_{7}^{2} \\
& -9 x_{7}^{4}-24 \sqrt{15} x_{4}^{2} x_{5} x_{7}+8 \sqrt{15} x_{5}^{3} x_{7} .
\end{aligned}
$$

Proof. Put $a_{4}=x_{1} e_{1}+x_{4} e_{4}+x_{5} e_{5}+x_{7} e_{7}, a_{1}=e_{2}$ and $a_{2}=c\left(x_{5} e_{4}-\right.$ $\left.x_{4} e_{5}-x_{7} e_{6}\right)\left(c=1 / \sqrt{x_{5}^{2}+x_{4}^{2}+x_{7}^{2}}\right)$. From Lemma 1 , we obtain an element

$$
g=\left(\begin{array}{ccccccc}
0 & 0 & 0 & x_{1} & 0 & 1 / c & 0 \\
1 & 0 & 0 & 0 & 0 & 0 & 0 \\
0 & 0 & 0 & 0 & -x_{1} & 0 & 1 / c \\
0 & c x_{5} & c x_{7} & x_{4} & 0 & -c x_{1} x_{4} & 0 \\
0 & -c x_{4} & 0 & x_{5} & -x_{7} & c x_{1} x_{5} & -c x_{1} x_{7} \\
0 & -c x_{7} & c x_{5} & 0 & x_{4} & 0 & c x_{1} x_{4} \\
0 & 0 & -c x_{4} & x_{7} & x_{5} & -c x_{1} x_{7} & c x_{1} x_{5}
\end{array}\right)
$$


of $G_{2}$ with $g \cdot e_{4}=a_{4}$. The vectors $v_{i}=g_{*}^{-1}\left(X_{i}(p)\right)$ are given as follows;

$$
\begin{aligned}
& v_{1}=\left(0,2 c\left(-x_{4}^{2}-x_{5}^{2}+3 x_{7}^{2}\right),-8 c x_{5} x_{7}, 0,-8 x_{4} x_{7}, 0,-8 c x_{1} x_{4} x_{7}\right), \\
& v_{2}=\left(-\sqrt{10} x_{4},-2 \sqrt{6} c x_{1} x_{4}, 0,0, \sqrt{10} x_{1} x_{5}-3 \sqrt{6} x_{1} x_{7}\right. \\
&\left.-2 \sqrt{6} c x_{5},-2 \sqrt{6} c x_{1}^{2} x_{7}+(1 / c)\left(-\sqrt{10} x_{5}+\sqrt{6} x_{7}\right)\right) \\
& v_{3}=\left(\sqrt{10} x_{5}+\sqrt{6} x_{7},-2 \sqrt{6} c x_{1} x_{5},-2 \sqrt{6} c x_{1} x_{7}, 0, \sqrt{10} x_{1} x_{4}\right. \\
&\left.2 \sqrt{6} c x_{4},-\sqrt{10}(1 / c) x_{4}\right) .
\end{aligned}
$$

Using the Mathematica we obtained the following

$$
\begin{aligned}
& \omega\left(v_{1}, v_{2}, v_{3}\right) \\
= & 24 \sqrt{15} x_{1} x_{4}^{3} x_{7}-24 \sqrt{15} c^{2} x_{1} x_{4}^{3} x_{7}+24 \sqrt{15} c^{2} x_{1}^{3} x_{4}^{3} x_{7}-40 \sqrt{15} x_{1} x_{4} x_{5}^{2} x_{7} \\
& +40 \sqrt{15} c^{2} x_{1} x_{4} x_{5}^{2} x_{7}-40 \sqrt{15} c^{2} x_{1}^{3} x_{4} x_{5}^{2} x_{7}+96 x_{1} x_{4} x_{5} x_{7}^{2}-96 c^{2} x_{1} x_{4} x_{5} x_{7}^{2} \\
& +96 c^{2} x_{1}^{3} x_{4} x_{5} x_{7}^{2}+24 \sqrt{15} x_{1} x_{4} x_{7}^{3}-24 \sqrt{15} c^{2} x_{1} x_{4} x_{7}^{3}+24 \sqrt{15} c^{2} x_{1}^{3} x_{4} x_{7}^{3} \\
& +\sqrt{-1}\left(-20 x_{4}^{4}-40 x_{4}^{2} x_{5}^{2}-20 x_{5}^{4}-64 \sqrt{15} x_{4}^{2} x_{5} x_{7}-32 \sqrt{15} c^{2} x_{4}^{2} x_{5} x_{7}\right. \\
& +32 \sqrt{15} c^{2} x_{1}^{2} x_{4}^{2} x_{5} x_{7}+32 \sqrt{15} c^{2} x_{5}^{3} x_{7}-32 \sqrt{15} c^{2} x_{1}^{2} x_{5}^{3} x_{7}+168 x_{4}^{2} x_{7}^{2} \\
& \left.+288 c^{2} x_{1}^{2} x_{4}^{2} x_{7}^{2}+72 x_{5}^{2} x_{7}^{2}+96 c^{2} x_{5}^{2} x_{7}^{2}+192 c^{2} x_{1}^{2} x_{5}^{2} x_{7}^{2}-36 x_{7}^{4}+288 c^{2} x_{1}^{2} x_{7}^{4}\right) .
\end{aligned}
$$

By a tedious calculation, we verified that the real part of the above vanishes and the imaginary part of the above reduces to $f\left(x_{1}, x_{4}, x_{5}, x_{7}\right)$.

Remark 7. Put $g\left(x_{1}, x_{4}, x_{5}, x_{7}\right)=x_{1}^{2}+x_{4}^{2}+x_{5}^{2}+x_{7}^{2}-1$. It is easily verified that $f\left(x_{1}, x_{4}, x_{5}, x_{7}\right)=g\left(x_{1}, x_{4}, x_{5}, x_{7}\right)=0$ hold at the point $\left(x_{1}, x_{4}, x_{5}, x_{7}\right)=( \pm 1 / 3,0,0, \pm 2 \sqrt{2} / 3)$ and the dimension of the orbit through $p=x_{1} e_{1}+x_{4} e_{4}+x_{5} e_{5}+x_{7} e_{7}$ is 3 . Furthermore, since the Jacobian $\partial(f, g) / \partial\left(x_{1}, x_{7}\right)$ is regular at the point $\left(x_{1}, x_{4}, x_{5}, x_{7}\right)$, there exist a 2-parameter family of 3-dimensional CR submanifolds.

\section{$\S 3 . \quad G e n e r a l i z a t i o n$ of Sekigawa's example}

\subsection{Sekigawa's example and its generalization}

In [13], Sekigawa obtained an example of 3-dimensional CR submanifold of $S^{6}$. His example was given as the image of the mapping of $S^{2} \times S^{1}$ into $S^{6}$

$$
\begin{aligned}
\Psi(y, t)= & \Psi\left(\left(y_{2}, y_{4}, y_{6}\right), e^{\sqrt{-1} t}\right) \\
= & \left(y_{2} \cos t\right) e_{2}-\left(y_{2} \sin t\right) e_{3}+\left(y_{4} \cos 2 t\right) e_{4}+\left(y_{4} \sin 2 t\right) e_{5} \\
& +\left(y_{6} \cos t\right) e_{6}+\left(y_{6} \sin t\right) e_{7}
\end{aligned}
$$


where $\left(y_{2}, y_{4}, y_{6}\right) \in S^{2}$ and $e^{\sqrt{-1} t} \in S^{1}$.

For a real triple $p=\left(p_{1}, p_{2}, p_{3}\right)$ with $p_{1}+p_{2}+p_{3}=0$ and $p_{1} p_{2} p_{3} \neq 0$, define a mapping $\psi_{p}$ of $S^{2} \times R$ to $S^{5} \subset S^{6}$ as follows;

$$
\begin{aligned}
& \psi_{p}\left(x_{1}, x_{2}, x_{3}, t\right) \\
= & \exp \left(t\left(p_{1} G_{51}+p_{2} G_{62}+p_{3} G_{73}\right)\right)\left(x_{1} e_{1}+x_{2} e_{2}+x_{3} e_{3}\right) \\
= & x_{1}\left(\cos \left(t p_{1}\right) e_{1}+\sin \left(t p_{1}\right) e_{5}\right)+x_{2}\left(\cos \left(t p_{2}\right) e_{2}+\sin \left(t p_{2}\right) e_{6}\right) \\
& +x_{3}\left(\cos \left(t p_{3}\right) e_{3}+\sin \left(t p_{3}\right) e_{7}\right),
\end{aligned}
$$

where $\left(x_{1}\right)^{2}+\left(x_{2}\right)^{2}+\left(x_{3}\right)^{2}=1$ and $t \in \mathbf{R}$. We use another expression;

$$
\psi_{p}\left(x_{1}, x_{2}, x_{3}, t\right)=\left(x_{1}, x_{2}, x_{3}\right) R_{p}(t),
$$

where $R_{p}(t)$ is the $\mathfrak{C}$-valued $(3,1)$-matrix

$$
R_{p}(t)=\left(\begin{array}{l}
\cos \left(t p_{1}\right) e_{1}+\sin \left(t p_{1}\right) e_{5} \\
\cos \left(t p_{2}\right) e_{2}+\sin \left(t p_{2}\right) e_{6} \\
\cos \left(t p_{3}\right) e_{3}+\sin \left(t p_{3}\right) e_{7}
\end{array}\right)
$$

It is easily seen that there exists an element $g \in G_{2}$ with $\Psi=g \circ \psi_{(2,-1,-1)}$.

The tangent space $d \psi_{\left(p_{1}, p_{2}, p_{3}\right)}\left(T_{x} S^{2} \oplus T_{t} \mathbf{R}\right)$ is generated by

$$
\begin{aligned}
d \psi_{p}((v, 0)) & =\left(v_{1}, v_{2}, v_{3}\right) R_{p}(t), \\
d \psi_{p}\left(\left(0, D_{t}\right)\right) & =\left(x_{1} p_{1}, x_{2} p_{2}, x_{3} p_{3}\right) R_{p}^{\prime}(t),
\end{aligned}
$$

where $v=\left(v_{1}, v_{2}, v_{3}\right)$ is a tangent vector of $S^{2}, D_{t}=\partial / \partial t$ is a tangent vector of $\mathbf{R}$ and

$$
R_{p}^{\prime}(t)=\left(\begin{array}{l}
-\sin \left(t p_{1}\right) e_{1}+\cos \left(t p_{1}\right) e_{5} \\
-\sin \left(t p_{2}\right) e_{2}+\cos \left(t p_{2}\right) e_{6} \\
-\sin \left(t p_{3}\right) e_{3}+\cos \left(t p_{3}\right) e_{7}
\end{array}\right)
$$

We can easily verify that

$$
\left\{\begin{array}{l}
\left\langle X R_{p}(t), Y R_{p}(t)\right\rangle=\left\langle X R_{p}^{\prime}(t), Y R_{p}^{\prime}(t)\right\rangle=\langle X, Y\rangle, \\
\left\langle X R_{p}(t), Y R_{p}^{\prime}(t)\right\rangle=0 .
\end{array}\right.
$$

hold for any $X, Y \in \mathbf{R}^{3}$. By a direct calculation, we have the following

LEMMA 8. The induced metric $\tilde{g}$ on $S^{2} \times \mathbf{R}$ is a warped product metric. Precisely

$$
\widetilde{g}=\pi_{1}^{*} g_{0}+\left(\sum_{i=1}^{3}\left(x_{i} p_{i}\right)^{2}\right) \pi_{2}{ }^{*} d t^{2}
$$

where $\pi_{1}: S^{2} \times \mathbf{R} \rightarrow S^{2}$ and $\pi_{2}: S^{2} \times \mathbf{R} \rightarrow \mathbf{R}$ are natural projections and $g_{0}$ is the canonical Riemannian metric on $S^{2}$. 
From (4), we have the following orthogonal direct sum decomposition

$$
\mathfrak{C}_{0}=V \oplus V^{\prime} \oplus \mathbf{R} e_{4}
$$

where we put

$$
V=\left\{X R_{p}(t): X \in \mathbf{R}^{3}\right\}, V^{\prime}=\left\{X R_{p}^{\prime}(t): X \in \mathbf{R}^{3}\right\}
$$

THEOREM 9. Let $p=\left(p_{1}, p_{2}, p_{3}\right)$ be a real triple with $p_{1}+p_{2}+p_{3}=0$ and $p_{1} p_{2} p_{3} \neq 0$. The image of the mapping

$$
\psi_{p}\left(x_{1}, x_{2}, x_{3}, t\right): S^{2} \times \mathbf{R} \rightarrow S^{5} \subset S^{6}
$$

is a 3-dimensional CR-submanifolds of $S^{6}$.

Proof. Let $x=\left(x_{1}, x_{2}, x_{3}\right)$ be an element of $S^{2}$ and $v=\left(v_{1}, v_{2}, v_{3}\right)$ be a tangent vector of $S^{2}$ at $x$. By direct calculation, we have

$$
\begin{aligned}
& J\left(d \psi_{p}((v, 0))\right) \\
= & \quad\left(v_{3} x_{2}-v_{2} x_{3}\right) \cos \left(p_{1} t\right) e_{1}+\left(-v_{3} x_{1}+v_{1} x_{3}\right) \cos \left(p_{2} t\right) e_{2} \\
& +\left(v_{2} x_{1}-v_{1} x_{2}\right) \cos \left(p_{3} t\right) e_{3}-\left(-v_{3} x_{2}+v_{2} x_{3}\right) \sin \left(p_{1} t\right) e_{5} \\
& -\left(v_{3} x_{1}-v_{1} x_{3}\right) \sin \left(p_{2} t\right) e_{6}-\left(-v_{2} x_{1}+v_{1} x_{2}\right) \sin \left(p_{3} t\right) e_{7} \\
= & (x \times v) R_{p}(t) .
\end{aligned}
$$

Thus we have $d \psi_{p}\left(T_{x} S^{2} \oplus\{0\}\right)$ is a $J$-invariant subspace. Since the image of the mapping $\psi_{p}$ is 3 -dimensional, we obtain the theorem.

For a non zero constant $k$ we can easily see

$$
\psi_{\left(k p_{1}, k p_{2}, k p_{3}\right)}(x, t)=\psi_{\left(p_{1}, p_{2}, p_{3}\right)}(x, k t) .
$$

Thus we may assume that $p_{3}=1$.

Remark 10. (1) If $p_{1} / p_{2}$ is a rational number, then $\psi_{\left(p_{1}, p_{2}, p_{3}\right)}$ is an immersion but not injective, and its image is a compact manifold.

(2) If $p_{1} / p_{2}$ is an irrational number, then $\psi_{\left(p_{1}, p_{2}, p_{3}\right)}$ is an injective immersion but not an embedding.

(3) Let $\tau$ be a permutation of 3 characters and put $p^{\prime}=\tau p$. There exists an element $g \in G_{2}$ such that $\psi_{p^{\prime}}=g \circ \psi_{p}$.

Next we shall calculate the second fundamental form of the immersion $\psi_{\left(p_{1}, p_{2}, p_{3}\right)} \cdot$ 
Lemma 11. For any $v, w \in T_{x} S^{2}, D_{t} \in T_{t} \mathbf{R}$ we have

(1) $\sigma(v, w)=0$

(2) $\sigma\left(D_{t}, D_{t}\right)=0$

$(3)$

$$
\begin{aligned}
\sigma(v, \xi) & \frac{1}{\sqrt{f(x)}}\left(v-\frac{1}{2} v(\log (f(x)) \cdot x)\left(\begin{array}{l}
p_{1}\left(-\sin \left(t p_{1}\right) e_{1}+\cos \left(t p_{1}\right) e_{5}\right) \\
p_{2}\left(-\sin \left(t p_{2}\right) e_{2}+\cos \left(t p_{2}\right) e_{6}\right) \\
p_{3}\left(-\sin \left(t p_{3}\right) e_{3}+\cos \left(t p_{3}\right) e_{7}\right)
\end{array}\right)\right.
\end{aligned}
$$

where $f(x)=\sum_{i=1}^{3}\left(x_{i} p_{i}\right)^{2}$ and $\xi=(1 / \sqrt{f(x)}) D_{t}$.

Proof. (1) is trivial, since the restriction of $\psi_{p}$ to $S^{2} \times\{t\}$ is a totally geodesic immersion for any $t \in \mathbf{R}$.

Let $\tilde{D}$ be the canonical connection of $\mathbf{R}^{7}$. From

$$
\tilde{D}_{D_{t}}\left(d \psi_{\left(p_{1}, p_{2}, p_{3}\right)}\left(0, D_{t}\right)\right)=-\left(x_{1} p_{1}{ }^{2}, x_{2} p_{2}{ }^{2}, x_{3} p_{3}{ }^{2}\right) R_{p}(t) \in V
$$

and $V=\mathbf{R} \psi(p, t) \oplus d \psi_{p}\left(T_{x} S^{2} \oplus\{0\}\right)$ we have (2).

For any tangent vector $v$ of $S^{2}$, we have

$$
\tilde{D}_{v}\left(d \psi_{\left(p_{1}, p_{2}, p_{3}\right)}\left(0, D_{t}\right)\right)=v\left(\begin{array}{l}
p_{1}\left(-\sin \left(t p_{1}\right) e_{1}+\cos \left(t p_{1}\right) e_{5}\right) \\
p_{2}\left(-\sin \left(t p_{2}\right) e_{2}+\cos \left(t p_{2}\right) e_{6}\right) \\
p_{3}\left(-\sin \left(t p_{3}\right) e_{3}+\cos \left(t p_{3}\right) e_{7}\right)
\end{array}\right)
$$

Taking the normal component, we get

$$
\begin{aligned}
& \sigma(v, \xi) \\
= & \left(\frac{1}{\sqrt{f(x)}}\right)\left\{\tilde{D}_{v}\left(d \psi_{\left(p_{1}, p_{2}, p_{3}\right)}\left(0, D_{t}\right)\right)-\left(\frac{v(f(x))}{2 f(x)}\right) d \psi_{\left(p_{1}, p_{2}, p_{3}\right)}\left(0, D_{t}\right)\right\} .
\end{aligned}
$$

From this proposition, we can calculate the trace and the square of the length of the second fundamental form. 


\section{Proposition 12.}

(1) Each immersion $\psi_{\left(p_{1}, p_{2}, p_{3}\right)}$ is a minimal immersion.

$(2)$

$$
|\sigma|^{2}=\frac{2}{\left(\sum_{i=1}^{3}\left(x_{i} p_{i}\right)^{2}\right)^{2}}\left\{\left(\sum_{i=1}^{3}\left(p_{i}\right)^{2}\right) \cdot\left(\sum_{i=1}^{3}\left(\left(x_{i} p_{i}\right)^{2}\right)-\left(\sum_{i=1}^{3}\left(x_{i}\right)^{2}\left(p_{i}\right)^{4}\right)\right\}\right.
$$

Since the scalar curvature $\tau\left(=6-|\sigma|^{2}\right)$ is not constant, we have the following

COROLlaRY 13. The induced metric is neither homogeneous nor cyclic parallel.

\section{REFERENCES}

[1] Bryant, R. L., Submanifolds and special structures on the octonians, J. Diff. Geometry, 17 (1982), 185-232.

[2] Dadok, J., Polar coordinates induced by actions of compact Lie groups, Trans. A. M. S., 288 (1985), 125-137.

[3] Dynkin, E.B., Semi-simple subalgebras of semi-simple Lie algebras, A.M.S.Transl. Ser. 2, 6 (1957), 111-244.

[4] Ejiri, N., Totally real submanifolds in a 6-sphere, Proc. A. M. S., 83 (1981), 759-763.

[5] — Equivariant minimal immersions of $S^{2}$ into $S^{2 m}(1)$, Trans. A. M. S., 297 (1986), 105-124.

[6] Freudenthal, H., Oktaven, Ausnahmegruppen und Oktavengeometrie, Geometriae Dedicata, 19 (1985), 7-63.

[7] Gray, A., Almost complex submanifolds of six sphere, Proc. A. M. S., 20 (1969), $277-279$.

[8] Harvey, R. and Lawson, H.B., Calibrated geometries, Acta Math., 148 (1982), 47-157.

[9] Hsiung, W. Y. and Lawson, H. B., Minimal submanifolds of low cohomogenity, J. Diff. Geometry, 5 (1971), 1-38.

[10] Mal'cev, A.I., On semi-simple subgroups of Lie groups, A.M.S. Transl, Ser. 1, 9 (1950), 172-213.

[11] Mashimo, K., Homogeneous totally real submanifolds of $S^{6}$, Tsukuba J. Math., 9 (1985), 185-202.

[12] Mashimo, K., Homogeneous CR submanifolds of $P^{3}(\mathbf{C})$, (in preparation).

[13] Sekigawa, K., Some CR-submanifolds in a 6-dimensioanl sphere, Tensor(N.S.), 6 (1984), 13-20. 
Hideya Hashimoto

Nippon Institute of Technology

4-1, Gakuendai, Miyashiro

Minami-Saitama Gun, Saitama 345-8501

Japan

hideya@nit.ac.jp

Katsuya Mashimo

Department of Mathematics

Tokyo University of Agriculture and Technology

Fuchu, Tokyo 183-0054

Japan

mashimo@cc.tuat.ac.jp 